\title{
On the discretization of Laine equations
}

Kostyantyn Zheltukhin, Natalya Zheltukhina

To cite this article: Kostyantyn Zheltukhin, Natalya Zheltukhina (2018) On the discretization of Laine equations, Journal of Nonlinear Mathematical Physics 25:1, 166177, DOI: https://doi.org/10.1080/14029251.2018.1440748

To link to this article: https://doi.org/10.1080/14029251.2018.1440748

Published online: 04 January 2021 


\title{
On the discretization of Laine equations
}

\author{
Kostyantyn Zheltukhin \\ Middle East Technical University, Department of Mathematics, \\ Universiteler Mahallesi, Dumlupinar Bulvar No:1, \\ 06800 Cankaya, Ankara, TURKEY \\ zheltukh@metu.edu.tr \\ Natalya Zheltukhina \\ Department of Mathematics, Faculty of Science, \\ Bilkent University, 06800 Bilkent, Ankara, Turkey \\ natalya@fen.bilkent.edu.tr
}

Received 20 July 2017

Accepted 31 July 2017

\begin{abstract}
We consider the discretization of Darboux integrable equations. For each of the integrals of a Laine equation we constructed either a semi-discrete equation which has that integral as an $n$-integral, or we proved that such an equation does not exist. It is also shown that all constructed semi-discrete equations are Darboux integrable.

Keywords: Semi-discrete chain; Darboux integrability; $x$-integral, $n$-integral; discretization.

2000 Mathematics Subject Classification: 35Q51, 37K60
\end{abstract}

\section{Introduction}

When considering hyperbolic type equations

$$
u_{x y}=g\left(x, y, u, u_{x}, u_{y}\right)
$$

one finds an important special subclass, so called Darboux integrable equations, that is described in terms of $x$ - and $y$-integrals. Recall that a function $W\left(x, y, u, u_{x}, u_{x x}, \ldots\right)$ is called a $y$-integral of equation (1.1) if $\left.D_{y} W\left(x, y, u, u_{x}, \ldots\right)\right|_{(1.1)}=0$, where $D_{y}$ represents the total derivative with respect to $y$ (see [2] and [8]). An $x$-integral $\bar{W}=\bar{W}\left(x, y, u, u_{y}, u_{y y}, \ldots\right)$ for equation (1.1) is defined in a similar way. Equation (1.1) is said to be Darboux integrable if it admits a nontrivial $x$-integral and a nontrivial $y$-integral.

The classification problem for Darboux integrable equations was considered by Goursat, Zhiber and Sokolov (see [2] and [8]). In his paper Goursat obtained a supposedly complete list of Darboux integrable equations of the form (1.1). A detailed discussion of the subject and corresponding references can be found in the survey [9].

Later Laine [7] published two Darboux integrable hyperbolic equations, which were absent in Goursat's list. The first equation found by Laine is

$$
u_{x y}=u_{x}\left(\frac{\sqrt{u_{y}}+u_{y}}{u-y}+\frac{u_{y}}{u-x}\right) .
$$


It has a second order $y$-integral

$$
W_{1}=\frac{u_{x x}}{u_{x}}-\frac{1}{2} u_{x}\left(\frac{1}{u-y}+\frac{3}{u-x}\right)+\frac{1}{u-x}
$$

and a third order $x$-integral

$$
\begin{aligned}
\bar{W}=\left(u_{y y y}-\frac{u_{y y}^{2}}{2 u_{y}}-u_{y y}\right. & \left.\frac{1+5 u_{y}^{\frac{1}{2}}+4 u_{y}}{u-y}\right)\left(u_{y y}-2 \frac{u_{y}+2 u_{y}^{\frac{3}{2}}+u_{y}^{2}}{u-y}\right)^{-1} \\
& -\left(\frac{2 u_{y}+2 u_{y}^{\frac{3}{2}}-6 u_{y}^{2}-10 u_{y}^{\frac{5}{2}}-4 u_{y}^{3}}{(u-y)^{2}}\right)\left(u_{y y}-2 \frac{u_{y}+2 u_{y}^{\frac{3}{2}}+u_{y}^{2}}{u-y}\right)^{-1} .
\end{aligned}
$$

The second equation found by Laine is

$$
u_{x y}=2\left((u+X)^{2}+u_{x}+(u+X) \sqrt{(u+X)^{2}+u_{x}}\right)\left(\frac{\sqrt{u_{y}}+u_{y}}{u-y}-\frac{u_{y}}{\sqrt{(u+X)^{2}+u_{x}}}\right) .
$$

It has a second order $y$-integral

$$
\begin{array}{r}
W_{2}=\frac{u_{x x}}{2 u_{x}}\left(1-\frac{u+X}{\sqrt{(u+X)^{2}+u_{x}}}\right)+u+\frac{(u+X)^{2}+2 u_{x}}{\sqrt{(u+X)^{2}+u_{x}}} \\
-\frac{(u+X)^{2}+u_{x}+(u+X) \sqrt{(u+X)^{2}+u_{x}}}{u-y}
\end{array}
$$

and a third order $x$-integral (1.4). For the second equation Laine assumed $X$ to be an arbitrary function of $x$. However Kaptsov (see [6]) has shown that $X$ must be a constant function if equation (1.5) admits the integrals (1.6) and (1.4). Thus it can be assumed, without loss of generality, that $X=0$.

One can also consider a semi-discrete analogue of Darboux integrable equations (see [1]). The notion of Darboux integrability for semi-discrete equations was developed by Habibullin (see [3]). For a function $t=t(n, x)$ of the continuous variable $x$ and discrete variable $n$ we introduce notations

$$
t_{k}=t(n+k, x), \quad k \in \mathbb{Z}, \quad t_{[m]}=\frac{d^{m}}{d x^{m}} t(n, x), \quad m \in \mathbb{N} .
$$

Then a hyperbolic type semi-discrete equation can be written as

$$
t_{1 x}=f\left(x, n, t, t_{1}, t_{x}\right) \text {. }
$$

A function $F$ of variables $x, n$, and $t, t_{1}, \ldots, t_{k}$ is called an $x$-integral of equation (1.7) if $\left.D_{x} F\right|_{(1.7)}=0$. A function $I$ of variables $x, n, t, t_{[1]}, \ldots, t_{[m]}$ is called an $n$-integral of equation (1.7) if $\left.D I\right|_{(1.7)}=I$, where $D$ is a shift operator. Equation (1.7) is said to be Darboux integrable if it admits a nontrivial $n$ integral and a nontrivial $x$-integral. In what follows we consider the equalities $D_{x} F=0$ and $D I=I$, which define $x$ - and $n$-integrals $F$ and $I$, only on solutions of the corresponding equations. For more information on semi-discrete Darboux integrable equations see [3], [4] and [5].

The interest in the continuous and discrete Darboux integrable models is stimulated by exponential type systems. Such systems are connected with semi-simple and affine Lie algebras which have applications in Liouville and conformal field theories. 
The discretization of equations from Goursat's list was considered by Habibullin and Zheltukhina in [5]. In the present paper we find semi-discrete versions of Laine equations (1.2) and (1.5). In particular we find semi-discrete equations that admit functions (1.3) or (1.6) as $n$-integrals, and show that these equations are Darboux integrable. This is the main result of our paper given in Theorem 1.1 and Theorem 1.2 below.

Theorem 1.1. The semi-discrete chain (1.7), which admits a minimal order n-integral

$$
I_{1}=\frac{t_{x x}}{t_{x}}-\frac{1}{2} t_{x}\left(\frac{1}{t-\varepsilon(n)}+\frac{3}{t-x}\right)+\frac{1}{t-x}
$$

where $\mathcal{E}(n)$ is an arbitrary function of $n$, is

$$
t_{1 x}=t_{x} \frac{\left(t_{1}-x\right)}{(t-x)} B\left(n, t, t_{1}\right)
$$

where $B$ is a function of $n, t, t_{1}$, satisfying the following equation

$$
\left(t_{1}-\varepsilon\right)\left(t_{1}-\varepsilon_{1}\right)-2(t-\varepsilon)\left(t_{1}-\varepsilon_{1}\right) B+(t-\varepsilon)\left(t-\varepsilon_{1}\right) B^{2}=0 .
$$

Moreover, chain (1.9) admits an x-integral of minimal order 3.

Theorem 1.2. The semi-discrete chain (1.7), which admits a minimal order n-integral

$$
I_{2}=\frac{t_{x x}}{2 t_{x}}\left(1-\frac{t}{\sqrt{t^{2}+t_{x}}}\right)+t+\frac{t^{2}+2 t_{x}}{\sqrt{t^{2}+t_{x}}}-\frac{t^{2}+t_{x}+t \sqrt{t^{2}+t_{x}}}{t-\varepsilon(n)},
$$

where $\varepsilon(n)$ is an arbitrary function of $n$, is

$$
t_{1 x}=2 A\left(t A-t_{1}\right) \sqrt{t^{2}+t_{x}}+A^{2} t_{x}+2 t A\left(t A-t_{1}\right)
$$

where $A$ is a function of $n, t, t_{1}$, satisfying the following system of equations

$$
\left\{\begin{array}{l}
A_{t}=\frac{-2 t_{1}\left(t_{1}-\varepsilon_{1}\right) A+(-\varepsilon+2 t)\left(t_{1}-\varepsilon_{1}\right) A^{2}-\varepsilon_{1}(t-2 \varepsilon) A^{3}}{2\left(t_{1}-\varepsilon_{1}\right)(t-\varepsilon)\left(t_{1}-t A\right)} \\
A_{t_{1}}=\frac{\varepsilon\left(t_{1}-\varepsilon_{1}\right)+(t-\varepsilon)\left(2 t_{1}-\varepsilon_{1}\right) A-2 t(t-2 \varepsilon) A^{2}}{2\left(t_{1}-\varepsilon_{1}\right)(t-\varepsilon)\left(t_{1}-t A\right)}
\end{array}\right.
$$

Moreover, chain (1.12) admits an x-integral of minimal order 2.

The paper is organized as follows. In Sections 2 and 3 we give proofs of Theorems 1.1 and 1.2 respectively. In Section 4 we show that function (1.4) can not be a minimal order $n$-integral for any equation (1.7). 


\section{Proof of Theorem 1.1}

Discretization by $n$-integral: Let us find $f\left(x, n, t, t_{1}, t_{x}\right)$ such that $D I_{1}=I_{1}$, where $I_{1}$ is defined by (1.8). Equality $D I_{1}=I_{1}$ implies

$$
\begin{aligned}
\frac{f_{x}+f_{t} t_{x}+f_{t_{1}} f+f_{t_{x}} t_{x x}}{f}-\frac{f}{2}\left(\frac{1}{t_{1}-\varepsilon_{1}}+\frac{3}{t_{1}-x}\right)+\frac{1}{t_{1}-x} & \\
& =\frac{t_{x x}}{t_{x}}-\frac{t_{x}}{2}\left(\frac{1}{t-\varepsilon}+\frac{3}{t-x}\right)+\frac{1}{t-x},
\end{aligned}
$$

where $\varepsilon=\varepsilon(n)$ and $\varepsilon_{1}=\varepsilon(n+1)$.

By comparing the coefficients before $t_{x x}$ in (2.1), we get $\frac{f_{t_{x}}}{f}=\frac{1}{t_{x}}$, which implies that $f=$ $A\left(x, n, t, t_{1}\right) t_{x}$. We substitute this expression for $f$ in (2.1) and get

$$
\begin{aligned}
\frac{A_{x}+A_{t} t_{x}+A_{t_{1}} A t_{x}}{A}-\frac{A t_{x}}{2}\left(\frac{1}{t_{1}-\varepsilon_{1}}+\frac{3}{t_{1}-x}\right)+\frac{1}{t_{1}-x} & \\
& =-\frac{t_{x}}{2}\left(\frac{1}{t-\varepsilon}+\frac{3}{t-x}\right)+\frac{1}{t-x} .
\end{aligned}
$$

The above equation is equivalent to a system of two equations

$$
\left\{\begin{array}{l}
\frac{A_{x}}{A}+\frac{1}{t_{1}-x}=\frac{1}{t-x}, \\
\frac{A_{t}}{A}+A_{t_{1}}-\frac{A}{2}\left(\frac{1}{t_{1}-\varepsilon_{1}}+\frac{3}{t_{1}-x}\right)=\frac{-1}{2}\left(\frac{1}{t-\varepsilon}+\frac{3}{t-x}\right) .
\end{array}\right.
$$

The first equation of system (2.3) can be written as $\frac{\partial}{\partial x}\left(\ln |A|-\ln \left|t_{1}-x\right|+\ln |t-x|\right)=0$ which implies that

$$
A\left(x, n, t, t_{1}\right)=\frac{t_{1}-x}{t-x} B\left(n, t, t_{1}\right)
$$

for some function $B$ of variables $n, t, t_{1}$. Substituting expression (2.4) for $A$ into the second equation of system (2.3), we get

$$
-\frac{1}{t-x}+\frac{B_{t}}{B}+\frac{B}{t-x}+\frac{B_{t_{1}}\left(t_{1}-x\right)}{t-x}-\frac{B\left(t_{1}-x\right)}{2(t-x)}\left(\frac{1}{t_{1}-\varepsilon_{1}}+\frac{3}{t_{1}-x}\right)=-\frac{1}{2}\left(\frac{1}{t-\varepsilon}+\frac{3}{t-x}\right) .
$$

Thus

$$
(t-x) \frac{B_{t}}{B}+\left(t_{1}-x\right) B_{t_{1}}-\frac{B}{2}\left(1+\frac{t_{1}-x}{t_{1}-\varepsilon_{1}}\right)=-\frac{1}{2}\left(1+\frac{t-x}{t-\varepsilon}\right) .
$$

We compare the coefficients before $x$ and $x^{0}$ in (2.6) and obtain

$$
\left\{\begin{array}{l}
-\frac{B_{t}}{B}-B_{t_{1}}+\frac{B}{2\left(t_{1}-\varepsilon_{1}\right)}=\frac{1}{2(t-\varepsilon)}, \\
\frac{t B_{t}}{B}+t_{1} B_{t_{1}}-\frac{B}{2}-\frac{t_{1} B}{2\left(t_{1}-\varepsilon_{1}\right)}=\frac{-1}{2}-\frac{t}{2(t-\varepsilon)},
\end{array}\right.
$$


which is equivalent to

$$
\left\{\begin{array}{l}
B_{t}=\frac{B\left(\varepsilon-2 t+t_{1}-\varepsilon B+t B\right)}{2(t-\varepsilon)\left(t-t_{1}\right)}, \\
B_{t_{1}}=\frac{-\varepsilon_{1}+t_{1}+\varepsilon_{1} B+t B-2 t_{1} B}{2\left(t_{1}-\varepsilon_{1}\right)\left(t-t_{1}\right)} .
\end{array}\right.
$$

The last system is compatible, that is $B_{t t_{1}}=B_{t_{1}}$, if and only if equality (1.10) is satisfied.

Existence of an $x$-integral: Let us show that equation (1.9) where function $B$ satisfies (1.10) has a finite dimensional $x$-ring. We have,

$$
t_{1 x}=\frac{t_{1}-x}{t-x} B t_{x}, \quad t_{2 x}=\frac{t_{2}-x}{t-x} B B_{1} t_{x}, \quad \text { and } \quad t_{3 x}=\frac{t_{3}-x}{t-x} B B_{1} B_{2} t_{x}
$$

where $B=B\left(n, t, t_{1}\right), B_{1}=B\left(n+1, t_{1}, t_{2}\right)$ and $B_{2}=B\left(n+2, t_{2}, t_{3}\right)$. We are looking for a function $F\left(x, n, t, t_{1}, t_{2}, t_{3}\right)$ such that $D_{x} F=0$, that is

$$
F_{x}+F_{t} t_{x}+F_{t_{1}} t_{1 x}+F_{t_{2}} t_{2 x}+F_{t_{3}} t_{3 x}=0 .
$$

Thus

$$
F_{x}+F_{t} t_{x}+F_{t_{1}} \frac{t_{1}-x}{t-x} B t_{x}+F_{t_{2}} \frac{t_{2}-x}{t-x} B B_{1} t_{x}+F_{t_{3}} \frac{t_{3}-x}{t-x} B B_{1} B_{2} t_{x}=0,
$$

which is equivalent to

$$
\left\{\begin{array}{l}
F_{x}=0 \\
(t-x) F_{t}+\left(t_{1}-x\right) B F_{t_{1}}+\left(t_{2}-x\right) B B_{1} F_{t_{2}}+\left(t_{3}-x\right) B B_{1} B_{2} F_{t_{3}}=0 .
\end{array}\right.
$$

By comparing the coefficients of $x^{0}$ and $x$ in the last equality we get the following system

$$
\left\{\begin{array}{l}
t F_{t}+t_{1} B F_{t_{1}}+t_{2} B B_{1} F_{t_{2}}+t_{3} B B_{1} B_{2} F_{t_{3}}=0 \\
-F_{t}-B F_{t_{1}}-B B_{1} F_{t_{2}}-B B_{1} B_{2} F_{t_{3}}=0
\end{array}\right.
$$

After diagonalization this system becomes

$$
\left\{\begin{array}{c}
F_{t}+\frac{B B_{1}\left(t_{2}-t_{1}\right)}{t-t_{1}} F_{t_{2}}+\frac{B B_{1} B_{2}\left(t_{3}-t_{1}\right)}{t-t_{1}} F_{t_{3}}=0 \\
F_{t_{1}}+\frac{B_{1}\left(t-t_{2}\right)}{t-t_{1}} F_{t_{2}}+\frac{B_{1} B_{2}\left(t-t_{3}\right)}{t-t_{1}} F_{t_{3}}=0 .
\end{array}\right.
$$

We introduce vector fields

$$
\begin{aligned}
& V_{1}=\frac{\partial}{\partial t}+\frac{B B_{1}\left(t_{2}-t_{1}\right)}{t-t_{1}} \frac{\partial}{\partial t_{2}}+\frac{B B_{1} B_{2}\left(t_{3}-t_{1}\right)}{t-t_{1}} \frac{\partial}{\partial t_{3}}, \\
& V_{2}=\frac{\partial}{\partial t_{1}}+\frac{B_{1}\left(t-t_{2}\right)}{t-t_{1}} \frac{\partial}{\partial t_{2}}+\frac{B_{1} B_{2}\left(t-t_{3}\right)}{t-t_{1}} \frac{\partial}{\partial t_{3}} .
\end{aligned}
$$

and $V=\left[V_{1}, V_{2}\right]$. Then, we have

$$
\frac{2\left(t-t_{1}\right)^{2}}{B_{1}} V=\left(t_{1}-t_{2}+B\left(t_{2}-t+\left(t-t_{1}\right) B_{1}\right) \frac{\partial}{\partial t_{2}}+B_{2}\left(t_{1}-t_{3}+B\left(t_{3}-t+\left(t-t_{1}\right) B_{1} B_{2}\right)\right) \frac{\partial}{\partial t_{3}}\right. \text {. }
$$


Direct calculation show that

$$
\left[V_{1}, V\right]=\frac{3 \varepsilon-4 t+t_{1}}{2(\varepsilon-t)\left(t-t_{1}\right)} V \quad \text { and } \quad\left[V_{2}, V\right]=\frac{3 \varepsilon_{1}+t-4 t_{1}}{2\left(\varepsilon_{1}-t_{1}\right)\left(t_{1}-t\right)} V .
$$

Hence vector fields $V_{1}, V_{2}$ and $V$ form a finite-dimensional ring. By the Jacobi Theorem the system of three equations $V_{1}(F)=0, V_{2}(F)=0, V(F)=0$ has a nonzero solution $F\left(t, t_{1}, t_{2}, t_{3}\right)$. The function $F\left(t, t_{1}, t_{2}, t_{3}\right)$ is an $x$-integral of equation (1.9).

\section{Proof of Theorem 1.2}

Discretization by $n$-integral: Let us find a function $f\left(x, n, t, t_{1}, t_{x}\right)$ such that $D I_{2}=I_{2}$, where $I_{2}$ is given by (1.11). The equality $D I_{2}=I_{2}$ implies that

$$
\begin{gathered}
\frac{f_{x}+f_{t} t_{x}+f_{t_{1}} f+f_{t_{x}} t_{x x}}{2 f}\left(1-\frac{t_{1}}{\sqrt{t_{1}^{2}+f}}\right)-\frac{t_{1}^{2}+f+t_{1} \sqrt{t_{1}^{2}+f}}{t_{1}-\varepsilon_{1}}+t_{1}+\frac{t_{1}^{2}+2 f}{\sqrt{t_{1}^{2}+f}} \\
=\frac{t_{x x}}{2 t_{x}}\left(1-\frac{t}{\sqrt{t^{2}+t_{x}}}\right)-\frac{t^{2}+t_{x}+t \sqrt{t^{2}+t_{x}}}{t-\varepsilon}+t+\frac{t^{2}+2 t_{x}}{\sqrt{t^{2}+t_{x}}},
\end{gathered}
$$

where $\varepsilon=\varepsilon(n)$ and $\varepsilon_{1}=\varepsilon(n+1)$. Comparing the coefficients before $t_{x x}$ in equality (3.1), we get

$$
\frac{f_{t_{x}}}{f}\left(1-\frac{t_{1}}{\sqrt{t_{1}^{2}+f}}\right)=\frac{1}{t_{x}}\left(1-\frac{t}{\sqrt{t^{2}+t_{x}}}\right) \text {. }
$$

This can be written as

$$
\frac{\partial}{\partial t_{x}} \ln \left(f \frac{\sqrt{f+t_{1}^{2}}+t_{1}}{\sqrt{f+t_{1}^{2}}-t_{1}}\right)=\frac{\partial}{\partial t_{x}} \ln \left(t_{x} \frac{\sqrt{t_{x}+t^{2}}+t}{\sqrt{t_{x}+t^{2}}-t}\right) .
$$

Thus

$$
\sqrt{f+t_{1}^{2}}+t_{1}=\left(\sqrt{t_{x}+t^{2}}+t\right) A\left(x, n, t, t_{1}\right)
$$

where $A$ is some function of variables $x, n, t$ and $t_{1}$. The last equality is equivalent to

$$
f=\left(2 A^{2} t-2 A t_{1}\right) \sqrt{t_{x}+t^{2}}+A^{2} t_{x}+t\left(2 A^{2} t-2 A t_{1}\right) .
$$

We substitute $f$ given by (3.5) into equality (3.1), use (3.4) and equality

$$
\sqrt{f+t_{1}^{2}}-t_{1}=\frac{f\left(\sqrt{t_{x}+t^{2}}-t\right)}{A t_{x}}
$$

to get

$$
\frac{1}{\sqrt{t_{x}+t^{2}} \sqrt{f+t_{1}^{2}}}\left(\Lambda_{1} t_{x}^{2}+\Lambda_{2} t_{x} \sqrt{t_{x}+t^{2}}+\Lambda_{3} t_{x}+\Lambda_{4} \sqrt{t_{x}+t^{2}}++\Lambda_{5} t^{2}\right)=0
$$

where

$$
\Lambda_{i}=\alpha_{i 1} A_{x}+\alpha_{i 2} A_{t}+\alpha_{i 3} A_{t_{1}}+\alpha_{i 4}, \quad 1 \leq i \leq 5
$$


and

$$
\begin{gathered}
\alpha_{11}=0, \alpha_{12}=1, \alpha_{13}=A^{2}, \alpha_{14}=\frac{A}{t-\varepsilon}-\frac{A^{3}}{t_{1}-\varepsilon_{1}}, \\
\alpha_{21}=0, \alpha_{22}=t-\frac{t_{1}}{A}, \alpha_{23}=-3 t_{1} A+3 t A^{2}, \alpha_{24}=\frac{-t_{1}+2 t A}{t-\varepsilon}+\frac{2 t_{1} A^{2}-3 t A^{3}}{t_{1}-\varepsilon_{1}}+A^{2}-A, \\
\alpha_{31}=1, \alpha_{32}=t^{2}, \alpha_{33}=2 t_{1}^{2}+5 t^{2} A^{2}-6 t_{1} t A, \\
\alpha_{34}=\frac{-t_{1} t+t(t+2 \varepsilon) A}{t-\varepsilon}+\frac{-5 t^{2} A^{3}+4 t_{1} t A^{2}-t_{1}^{2} A}{t_{1}-\varepsilon_{1}}+t_{1}+2 t A^{2}-t_{1} A, \\
\alpha_{41}=t-\frac{t_{1}}{A}, \alpha_{42}=0, \alpha_{43}=4 t^{3} A^{2}-6 t_{1} t^{2} A+2 t_{1}^{2} t, \\
\alpha_{44}=\frac{2 \varepsilon t^{2} A+\varepsilon t t_{1}}{t-\varepsilon}+\frac{-4 t^{3} A^{3}+4 t_{1} t^{2} A^{2}-t_{1}^{2} t A}{t_{1}-\varepsilon_{1}}+2 t^{2} A^{2}-t_{1} t A, \\
\alpha_{51}=1, \alpha_{52}=0, \alpha_{53}=2 t_{1}^{2}+4 t^{2} A^{2}-6 t_{1} t A, \\
\alpha_{54}=\frac{-t_{1} t+2 \varepsilon t}{t-\varepsilon}+\frac{-4 t^{2} A^{3}+4 t_{1} t A^{2}-t_{1}^{2} A}{t_{1}-\varepsilon_{1}}+t_{1}+2 t A^{2}-t_{1} A .
\end{gathered}
$$

We can solve the overdetermined system of linear equations $\Lambda_{i}=0, i=1,2 \ldots 5$, with respect to $A_{x}$, $A_{t}, A_{t_{1}}$ and obtain

$$
\left\{\begin{array}{l}
A_{x}=0 \\
A_{t}=-\frac{A}{t-\varepsilon}+\frac{A^{2}}{2\left(t_{1}-t A\right)}\left(\frac{A \varepsilon_{1}}{t_{1}-\varepsilon_{1}}-\frac{\varepsilon}{t-\varepsilon}\right) \\
A_{t_{1}}=\frac{A}{t_{1}-\varepsilon_{1}}-\frac{1}{2\left(t_{1}-t A\right)}\left(\frac{A \varepsilon_{1}}{t_{1}-\varepsilon_{1}}-\frac{\varepsilon}{t-\varepsilon}\right) .
\end{array}\right.
$$

By direct calculations one can check that $A_{t t_{1}}=A_{t_{1}}$, so the above system has a solution.

Existence of an $x$-integral: We are looking for a function $F\left(t, t_{1}, t_{2}\right)$ such that $D_{x} F=0$ that is

$$
F_{t} t_{x}+F_{t_{1}} t_{1 x}+F_{t_{2}} t_{2 x}=0
$$

where $t$ satisfies equation (1.7) with function $f$ given by (3.5). We use

$$
t_{1 x}=A^{2}\left(t, t_{1}\right) t_{x}+2 A\left(t, t_{1}\right)\left(t A\left(t, t_{1}\right)-t_{1}\right)\left(\sqrt{t_{x}+t^{2}}+t\right)
$$

and

$$
\sqrt{f+t_{1}^{2}}=\left(\sqrt{t_{x}+t^{2}}+t\right) A-t_{1}
$$


to get

$$
\begin{gathered}
t_{2 x}=A^{2}\left(t, t_{1}\right) A^{2}\left(t_{1}, t_{2}\right) t_{x}+2\left(\sqrt{t_{x}+t^{2}}+t\right)\left(t A\left(t, t_{1}\right)-t_{1}\right) A\left(t, t_{1}\right) A^{2}\left(t_{1}, t_{2}\right)+ \\
2\left(\sqrt{t_{x}+t^{2}}+t\right)\left(t_{1} A\left(t_{1}, t_{2}\right)-t_{2}\right) A\left(t, t_{1}\right) A\left(t_{1}, t_{2}\right) .
\end{gathered}
$$

By substituting these expressions for $t_{1 x}$ and $t_{2 x}$ into equality (3.9) and comparing the coefficients of $\sqrt{t_{x}+t^{2}}, t_{x}$ and $t_{x}^{0}$, we obtain the following system of equations

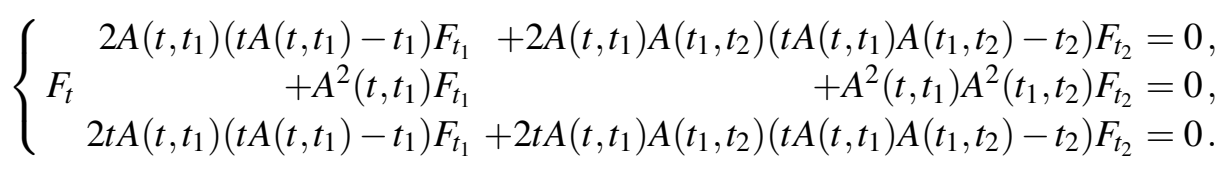

To check for the existence of a solution we transform the above system to its row reduced form

$$
\left\{\begin{array}{c}
F_{t}+\frac{A^{2}\left(t, t_{1}\right) A\left(t_{1}, t_{2}\right)\left(t_{2}-t_{1} A\left(t_{1}, t_{2}\right)\right)}{t A\left(t, t_{1}\right)-t_{1}} F_{t_{2}}=0, \\
F_{t_{1}}+\frac{A\left(t_{1}, t_{2}\right)\left(t_{2}-t A\left(t, t_{1}\right) A\left(t_{1}, t_{2}\right)\right)}{-t A\left(t, t_{1}\right)+t_{1}} F_{t_{2}}=0 .
\end{array}\right.
$$

The corresponding vector fields

$$
\begin{gathered}
V_{1}=\frac{\partial}{\partial t}+\frac{A^{2}\left(t, t_{1}\right) A\left(t_{1}, t_{2}\right)\left(t_{2}-t_{1} A\left(t_{1}, t_{2}\right)\right)}{t A\left(t, t_{1}\right)-t_{1}} \frac{\partial}{\partial t_{2}}, \\
V_{2}=\frac{\partial}{\partial t_{1}}+\frac{A\left(t_{1}, t_{2}\right)\left(t_{2}-t A\left(t, t_{1}\right) A\left(t_{1}, t_{2}\right)\right)}{-t A\left(t, t_{1}\right)+t_{1}} \frac{\partial}{\partial t_{2}}
\end{gathered}
$$

commute, that is $\left[V_{1}, V_{2}\right]=0$, provided $A$ satisfies system (3.8). Thus by the Jacobi theorem, system (3.10) has a solution. To solve the system define a function $E\left(t, t_{1}, t_{2}\right)$ by

$$
E_{t}=\frac{A^{2}}{t A-t_{1}}, E_{t_{2}}=\frac{1}{A_{1}\left(t_{1} A_{1}-t_{2}\right)}, E_{t_{1}}=\frac{t_{2}-t A A_{1}}{\left(t A-t_{1}\right)\left(t_{1} A_{1}-t_{2}\right)}+\frac{1}{t_{1}-\varepsilon_{1}} E
$$

where $A=A\left(t, t_{1}\right)$ and $A_{1}=A\left(t_{1}, t_{2}\right)$.

One can check that $E_{t t_{1}}=E_{t_{1} t}$ and $E_{t_{1} t_{2}}=E_{t_{2} t_{1}}$, so such a function $E$ exists. Function $E$ is a first integral of the first equation of system (3.10). We write system (3.10) using new variables

$$
\tilde{t}=t, \tilde{t}_{1}=t_{1}, \tilde{t}_{2}=E\left(t, t_{1}, t_{2}\right)
$$

and obtain

$$
\left\{\begin{array}{l}
F_{\tilde{t}}=0 \\
F_{\tilde{t}_{1}}+\frac{\tilde{t}_{2}}{\tilde{t}_{1}-\varepsilon_{1}} F_{\tilde{t}_{2}}=0
\end{array}\right.
$$

Therefore one of the $x$-integrals is $F\left(t, t_{1}, t_{2}\right)=E\left(t, t_{1}, t_{2}\right) /\left(t_{1}-\varepsilon(n+1)\right)$ where function $E$ defined above. 


\section{Nonexistence of a chain (1.7) admitting the minimal order $n$-integral (1.4)}

Let us find a function $f\left(x, n, t, t_{1}, t_{x}\right)$ such that equation (1.7) has the $n$-integral

$$
I=\frac{t_{x x x}-\frac{t_{x x}^{2}}{2 t_{x}}-t_{x x} \frac{1+5 \sqrt{t_{x}}+4 t_{x}}{t-x}-\frac{2 t_{x}+2 t_{x} \sqrt{t_{x}}-6 t_{x}^{2}-10 t_{x}^{2} \sqrt{t_{x}}-4 t_{x}^{3}}{(t-x)^{2}}}{t_{x x}-\frac{2 t_{x}+4 t_{x} \sqrt{t_{x}}+2 t_{x}^{2}}{t-x}} .
$$

We have,

$$
\begin{gathered}
t_{1 x}=f\left(x, n, t, t_{1}, t_{x}\right), \\
t_{1 x x}=f_{x}+f_{t} t_{x}+f_{t_{1}} f+f_{t_{x}} t_{x x} \\
t_{1 x x x}=\left(f_{x x}+f_{x t} t_{x}+f_{x t_{1}} f+f_{x t_{x}} t_{x x}\right)+t_{x}\left(f_{x t}+f_{t t} t_{x}+f_{t t_{1}} f+f_{t t_{x}} t_{x x}\right)+f_{t} t_{x x} \\
+f\left(f_{x t_{1}}+f_{t t_{1}} t_{x}+f_{t_{1} t_{1}} f+f_{t_{1} t_{x}} t_{x x}\right)+f_{t_{1}}\left(f_{x}+f_{t} t_{x}+f_{t_{1}} f+f_{t_{x}} t_{x x}\right) \\
+t_{x x}\left(f_{x t_{x}}+f_{t t_{x}} t_{x}+f_{t_{1} t_{x}} f+f_{t_{x} t_{x}} t_{x x}\right)+f_{t_{x}} t_{x x x} .
\end{gathered}
$$

Equality $D I=I$ is equivalent to $J:=L(D L)(D I-I)=0$, where $L=\sqrt{2} t_{x}(t-x)\left\{t_{x x}(t-x)-\right.$ $\left.2 t_{x}\left(\sqrt{t_{x}}+1\right)^{2}\right\}$. We have,

$$
J=\Lambda_{1} t_{x x x}+\Lambda_{2} t_{x x}^{3}+\Lambda_{3} t_{x x}^{2}+\Lambda_{4} t_{x x}+\Lambda_{5},
$$

where $\Lambda_{k}, 1 \leq k \leq 5$, are some functions of variables $x, n, t, t_{1}, t_{x}$. In particular,

$$
\begin{gathered}
\frac{\Lambda_{1}}{2(t-x)\left(t_{1}-x\right) t_{x} f}=2(t-x) f(1+\sqrt{f})^{2}-2\left(t_{1}-x\right) t_{x} f_{t_{x}}\left(1+\sqrt{t_{x}}\right)^{2}-\left(t_{1}-x\right)(t-x)\left(f_{x}+f_{t} t_{x}+f_{t_{1}} f\right), \\
\Lambda_{2}=(t-x)^{2}\left(t_{1}-x\right)^{2}\left\{f f_{t_{x}}-t_{x} f_{t_{x}}^{2}+2 t_{x} f f_{t_{x} t_{x}}\right\}, \\
\frac{\Lambda_{3}}{(t-x)\left(t_{1}-x\right)}=(t-x) f\left[4 f^{3 / 2}+2 f^{2}+\left(x-t_{1}\right) f_{x}+f\left(2+\left(x-t_{1}\right) f_{t_{1}}\right)\right]+10\left(x-t_{1}\right) t_{x}^{3 / 2} f f_{t_{x}} \\
+t_{x}\left[10(t-x) f^{3 / 2} f_{t_{x}}+2(t-x)\left(t_{1}-x\right) f_{t_{x}} f_{x}+4(t-x) f^{2}\left(2 f_{t_{x}}+\left(x-t_{1}\right) f_{t_{1} t_{x}}\right)\right] \\
+t_{x} f\left(2\left(t-t_{1}\right) f_{t_{x}}+(t-x)\left(x-t_{1}\right)\left(3 f_{t}+4 f_{x t_{x}}\right)\right) \\
-2\left(t_{1}-x\right) t_{x}^{2}\left[2 f\left(2 f_{t_{x}}-f_{t_{x} t_{x}}+(t-x) f_{t t_{x}}\right)+f_{t_{x}}\left(f_{t_{x}}+(x-t) f_{t}\right)\right] \\
-4\left(f_{t_{x}}^{2}-2 f f_{t_{x} t_{x}}\right)\left(t_{1}-x\right) t_{x}^{5 / 2}-2\left(f_{t_{x}}^{2}-2 f f_{t_{x} t_{x}}\right)\left(t_{1}-x\right) t_{x}^{3} .
\end{gathered}
$$

Equality $\Lambda_{2}=0$ implies that $f f_{t_{x}}-t_{x} f_{t_{x}}^{2}+2 t_{x} f f_{t_{x} t_{x}}=0$, thus

$$
\frac{f^{2}}{f_{t_{x}}} \frac{\partial}{\partial t_{x}}\left\{\frac{t_{x} f_{t_{x}}^{2}}{f}\right\}=0
$$


Hence, $\frac{t_{x} f_{t_{x}}^{2}}{f}=A^{2}\left(x, n, t, t_{1}\right)$ for some function $A$ depending on $x, n, t, t_{1}$ only. Therefore, $\frac{f_{t_{x}}}{\sqrt{f}}=\frac{A}{\sqrt{t_{x}}}$ and hence $\frac{\partial}{\partial t_{x}}\left\{\sqrt{f}-A \sqrt{t_{x}}=0\right\}$. We have,

$$
\sqrt{f}=A \sqrt{t_{x}}+B
$$

where $A=A\left(x, n, t, t_{1}\right)$ and $B=B\left(x, n, t, t_{1}\right)$. We substitute $f=A^{2} t_{x}+2 A B \sqrt{t_{x}}+B^{2}$ into $\Lambda_{1}=0$ and get

$$
\alpha_{1} t_{x}^{2}+\alpha_{2} t_{x}^{3 / 2}+\alpha_{3} t_{x}+\alpha_{4} \sqrt{t_{x}}+\alpha_{5}=0
$$

We solve the system of equations $\alpha_{k}=0,1 \leq k \leq 5$, and obtain $B=0$, that is

$$
\left\{\begin{array}{l}
A_{x}=\frac{B}{2 A} B_{t}-\frac{3}{2} A B B_{t_{1}}+\frac{2\left(t_{1}-x\right) B+A\left\{2\left(t-t_{1}\right)+6(t-x) B+3(t-x) B^{2}\right\}}{2(t-x)\left(x-t_{1}\right)} \\
A_{t}=\frac{A}{2 B} B_{t}+\frac{A^{3}}{2 B} B_{t_{1}}+\frac{A\left\{2\left(t_{1}-x\right) A+2\left(x-t_{1}\right) B-(t-x) A^{2}(2+B)\right\}}{2(t-x)\left(x-t_{1}\right) B} \\
A_{t_{1}}=-\frac{1}{2 A B} B_{t}-\frac{A}{2 B} B_{t_{1}}+\frac{2\left(x-t_{1}\right)+(t-x) A(2+3 B)}{2(t-x)\left(x-t_{1}\right) B} \\
B_{x}=-B^{2} B_{t_{1}}-\frac{B(1+B)^{2}}{t_{1}-x}
\end{array}\right.
$$

We substitute $f=A^{2} t_{x}+2 A B \sqrt{t_{x}}+B^{2}$ into $\Lambda_{3}=0$ and get

$$
\beta_{1} t_{x}^{3}+\beta_{2} t_{x}^{5 / 2}+\beta_{3} t_{x}^{2}+\beta_{4} t_{x}^{3 / 2}+\beta_{5} t_{x}+\beta_{6} \sqrt{t_{x}}+\beta_{7}=0 .
$$

We solve the system of equations $\beta_{k}=0,1 \leq k \leq 7$, and obtain $B=0$, or

$$
\left\{\begin{array}{l}
A_{x}=\frac{3 B}{8 A} B_{t}-\frac{23}{24} A B B_{t_{1}}+\frac{21\left(t_{1}-x\right) B+A\left\{16\left(t-t_{1}\right)+51(t-x) B+23(t-x) B^{2}\right\}}{24(t-x)\left(x-t_{1}\right)} \\
A_{t}=\frac{3 A}{8 B} B_{t}+\frac{3 A^{3}}{8 B} B_{t_{1}}+\frac{A\left\{7\left(t_{1}-x\right) A+8\left(x-t_{1}\right) B-(t-x) A^{2}(7+3 B)\right\}}{8(t-x)\left(x-t_{1}\right) B} \\
A_{t_{1}}=-\frac{3}{8 A B} B_{t}-\frac{3 A}{8 B} B_{t_{1}}+\frac{7\left(x-t_{1}\right)+(t-x) A(7+11 B)}{8(t-x)\left(x-t_{1}\right) B} \\
B_{x}=-B^{2} B_{t_{1}}-\frac{B(1+B)^{2}}{t_{1}-x}
\end{array}\right.
$$

We equate expressions for $A_{x}$ and $A_{t}$ from (4.1) and (4.2) and find

$$
\left\{\begin{array}{l}
B_{t}=-\frac{A\left\{2\left(t_{1}-x\right) B+A\left(\left(t-t_{1}\right)+(t-x) B\right)\right\}}{2(t-x)\left(x-t_{1}\right) B} \\
B_{t_{1}}=\frac{t-t_{1}+3(t-x) B+2(t-x) B^{2}}{2(t-x)(x-t-1) B}
\end{array}\right.
$$


Then, it follows from (4.1) that

$$
\left\{\begin{array}{l}
A_{x}=\frac{\left(t_{1}-x+(t-x) A\right) B}{2(t-x)\left(x-t_{1}\right)} \\
A_{t}=\frac{A\left(\left(t_{1}-x\right) A+(x-t) A^{2}+2\left(x-t_{1}\right) B\right)}{2(t-x)\left(x-t_{1}\right) B} \\
A_{t_{1}}=\frac{x-t_{1}+(t-x) A(1+2 B)}{2(t-x)\left(x-t_{1}\right) B} \\
B_{x}=\frac{B\left(t+t_{1}-2 x+(t-x) B\right)}{2\left(t_{1}-x\right)(x-t)}
\end{array}\right.
$$

Equality $A_{t t_{1}}-A_{t_{1} t}=0$ becomes $\frac{\left(t_{1}-x\right)^{2}-(t-x)^{2} A^{3}}{(t-x)^{2}\left(t_{1}-x\right)^{2} B}=0$, thus

$$
A^{3}=\frac{\left(t_{1}-x\right)^{2}}{(t-x)^{2}}
$$

Equality $A_{x t_{1}}-A_{t_{1} x}=0$ becomes $\frac{-\left(t_{1}-x\right)^{2}+(t-x)^{2} A(1+B)^{2}}{(t-x)^{2}\left(t_{1}-x\right)^{2} B}=0$, thus

$$
A(1+B)^{2}=\frac{\left(t_{1}-x\right)^{2}}{(t-x)^{2}} .
$$

Equality $A_{x t}-A_{t x}=0$ becomes $\frac{\left(t_{1}-x\right)^{2}(A-B)^{2}-(t-x)^{2} A^{3}}{(t-x)^{2}\left(t_{1}-x\right)^{2} B}=0$. It implies that

$$
\frac{A^{3}}{(A-B)^{2}}=\frac{\left(t_{1}-x\right)^{2}}{(t-x)^{2}}
$$

or $A=B$, that leads to $A=B=0$ and $f=0$. It follows from (4.5) and (4.7) that $A-B=1$ or $A-B=-1$. It follows from (4.5) and (4.6) that $1+B=A$ or $1+B=-A$. This gives rise to four possibilities:

1) $A-B=1$;

2) $A-B=1$ and $A+B=-1$ which gives $A=0, B=-1$ and therefore $f=1$;

3) $A-B=-1$ and $A-B=1$ which is an inconsistent system;

4) $A-B=-1$ and $A+B=-1$ which gives $A=-1, B=0$ and therefore $f=t_{x}$.

We have to study case 1) only. In this case we get $B=A-1$ and equation $\sqrt{t_{1 x}}=A \sqrt{t_{x}}+B$ becomes $\sqrt{t_{1 x}}+1=A\left(\sqrt{t_{x}}+1\right)$, that can be written as well as

$$
\left(\sqrt{t_{1 x}}+1\right)^{3}=A^{3}\left(\sqrt{t_{x}}+1\right)^{3} .
$$

Due to (4.5), our equation (4.8) becomes

$$
\frac{\left(\sqrt{t_{1 x}}+1\right)^{3}}{\left(t_{1}-x\right)^{2}}=\frac{\left(\sqrt{t_{x}}+1\right)^{3}}{(t-x)^{2}} .
$$

The last equation admits an $n$-integral $I=\frac{\left(\sqrt{t_{x}}+1\right)^{3}}{(t-x)^{2}}$ of order one. 
Let us consider case $B=0$. We write $D I-I=0$ for the chain $t_{1 x}=C\left(x, n, t, t_{1}\right) t_{x}$ and get

$$
\Lambda_{1} t_{x x x}+\Lambda_{2} t_{x x}^{2}+\Lambda_{3} t_{x x}+\Lambda_{4}=0
$$

where $\Lambda_{k}=\Lambda_{k}\left(x, n, t, t_{1}, t_{x}\right), 1 \leq k \leq 4$. Equation $\Lambda_{1}=0$ implies

$$
\alpha_{1} t_{x}+\alpha_{2} \sqrt{t_{x}}+\alpha_{3}=0
$$

where $\alpha_{k}=\alpha_{k}\left(x, n, t, t_{1}\right), 1 \leq k \leq 3$. In particular, $\alpha_{2}=4 C\left(-\left(t_{1}-x\right)+(t-x) \sqrt{C}\right)$. Since $\alpha_{2}=0$, we have $C=\left(t_{1}-x\right)^{2}(t-x)^{-2}$. The chain becomes $t_{1 x}=\left(t_{1}-x\right)^{2}(t-x)^{-2} t_{x}$. It admits the $n$-integral $I=(t-x)^{-2} t_{x}$ of order one.

Therefore, if equation (1.7) admits $n$-integral (1.4) then (1.4) is not a minimal order integral.

\section{Acknowledgment}

We are thankful to Prof. Habibullin for suggesting the Laine equations discretization problem and for his interest in our work.

\section{References}

[1] V. E. Adler, S.Ya. Startsev, On discrete analogues of the Liouville equation, Theoret. and Math. Phys. 121(2) (1999) 1484-1495.

[2] E. Goursat, Recherches sur quelques équations aux dérivés partielles du second ordre, Annales de la faculté des Sciences de l'Université de Toulouse 2e série 1(1) (1899) 31-78.

[3] I. Habibullin, A. Pekcan, Characteristic Lie Algebra and Classification of Semi-Discrete Models, Theoret. and Math. Phys. 151(3) (2007) 781-790.

[4] I. T. Habibullin, N. Zheltukhina, and A. Sakieva, Discretization of hyperbolic type Darboux integrable equations preserving integrability, J. Math. Phys., 52(9) (2011), 093507.

[5] I. Habibullin, N. Zheltukhina, Discretization of Liouville type nonautonomous equations preserving integrals, Journal of Non-linear Mathematical Physics 23(4) (2016) 620-642.

[6] O. V. Kaptsov, On the Goursat classification problem, Program. Comput. Softw. 38(2) (2012) 102 104.

[7] M. E. Laine, Sur l'a application de la method de Darboux aux equations $s=f(x, y, z, p, q)$, Comptes rendus V.182 (1926) 1127-1128.

[8] A. V. Zhiber and V. V. Sokolov, Exactly integrable hyperbolic equations of Liouville type, Russian Mathematical Surveys 56(1) (2001) 61-101.

[9] A. V. Zhiber, R. D. Murtazina, I. T. Habibullin, A. B. Shabat, Characteristic Lie rings and integrable models in mathematical physics, Ufa Math. J., 4(3) (2012) 17-85. 